\title{
Multiplicity of solutions to a four-point boundary value problem of a differential system via variational approach
}

\author{
Weigao $\mathrm{Ge}^{1}$ and Zhihong Zhao ${ }^{2 *}$
}

"Correspondence: zzh@ustb.edu.cn
2Department of Mathematics,
University of Science and
Technology Beijing, Beijing, 100083,
P.R. China
Full list of author information is
available at the end of the article

\begin{abstract}
By constructing an adequate real functional and choosing an appropriate admissible function space, the existence of multiple solutions to a four-point boundary value problem, which may be taken as an extension of Sturm-Liouville boundary value problems, is proved via a variational approach for a second-order differential system with a p-Laplacian.
\end{abstract}

MSC: $34 \mathrm{~B} 15$

Keywords: four-point boundary value problem; functional; critical point; variational approach; differential system

\section{Introduction}

The variational approach, together with the critical point theory, is one of the important methods in the study of two-point boundary value problems of ordinary differential equation [1-5], as well as impulsive differential equations [6-9]. However, this approach is much more effective in the study of boundary value problems of differential systems [1012].

Mawhin and Willem [10] studied the existence of periodic solutions of convex Hamiltonian system in the form

$$
\left\{\begin{array}{l}
J u^{\prime}(t)+\nabla H(t, u(t))=0 \\
u(0)-u(T)=0
\end{array}\right.
$$

where $H:[0, T] \times \mathbb{R}^{2 n} \rightarrow \mathbb{R}$ and proved that the problem has at least one periodic solution if

$$
(l(t), u) \leq H(t, u) \leq \frac{\alpha}{2}|u|^{2}+\gamma(t), \quad \int_{0}^{T} H(t, u) \mathrm{d} t \rightarrow \infty, \quad \text { as }|u| \rightarrow \infty,
$$

with $\alpha \in\left(0, \frac{2 \pi}{T}\right)$ ([10], Theorem 3.1). Also, they proved the system

$$
\left\{\begin{array}{l}
\left(M(t, u) u^{\prime}(t)\right)^{\prime}-\frac{1}{2}\left(\nabla_{u}\left(M(t, u) u^{\prime}\right), u^{\prime}\right)+\nabla F(t, u)=f(t), \\
u(0)-u(T)=u^{\prime}(0)-u^{\prime}(T)=0,
\end{array}\right.
$$

\section{Springer}


has at least one periodic solution if $|F(t, u)|+|\nabla F(t, u)| \leq h(t),\left(M(t, u) u^{\prime}, u^{\prime}\right) \geq \alpha\left|u^{\prime}\right|^{2}$, and $M$ is $T_{i}$-periodic in $u_{i}([10]$, Theorem 4.3).

Tian and Ge [11] discussed the differential system with a p-Laplacian

$$
\left\{\begin{array}{l}
\frac{\mathrm{d}}{\mathrm{d} t} \varphi_{p}\left(u^{\prime}(t)\right)+\nabla F(t, u(t))=0 \\
u(0)-u(T)=u^{\prime}(0)-u^{\prime}(T)=0
\end{array}\right.
$$

where $\varphi_{p}(x)=|x|^{p-2} x$ for $x \in \mathbb{R}^{n}$, and they obtained an existence theorem of periodic solutions under the condition

$$
\left(l(t),|u|^{\frac{p-2}{2}} u\right) \leq F(t, u) \leq \frac{\alpha^{2}}{p}|u|^{p}+\gamma(t), \quad \int_{0}^{T} F(t, u) \mathrm{d} t \rightarrow \infty, \quad \text { as }|u| \rightarrow \infty .
$$

The result extended that given by Mawhin and Willem ([10], Theorem 3.5).

Graef et al. studied in [12] the existence of at least three classical solutions to the multipoint value system

$$
\left\{\begin{array}{l}
\left(\phi_{p}\left(u^{\prime}\right)\right)^{\prime}+\lambda F(t, u)+\mu G(t, u)=0, \quad 0<t<1, \\
u(0)=\sum_{j=1}^{m} a_{j} u\left(t_{j}\right), \quad u(1)=\sum_{j=1}^{m} b_{j} u\left(t_{j}\right),
\end{array}\right.
$$

where $\phi_{p}(s)=\left(\phi_{p_{1}}\left(s_{1}\right), \phi_{p_{2}}\left(s_{2}\right), \ldots, \phi_{p_{n}}\left(s_{n}\right)\right)$ with $\phi_{p_{k}}\left(s_{k}\right)=\left|s_{k}\right|^{p_{k}-2} s_{k}, p_{k}>1, a_{j}, b_{j} \in \mathbb{R}, F, G$ : $[0,1] \times \mathbb{R}^{n} \rightarrow \mathbb{R}, \lambda, \mu>0$. By use of the existence theorem of three critical points given by Ricceri [13], they obtained sufficient conditions for the existence of three solutions to the discussed system, when the parameter $\lambda$ is defined in a certain interval $[0, \delta]$.

In this paper, we are to study the existence of multiple solutions to the following fourpoint boundary value problem (BVP for short):

$$
\left\{\begin{array}{l}
\left(P(t) x^{\prime}\right)^{\prime}=\nabla F(t, x), \quad 0<t<1 \\
x^{\prime}(0)=\alpha x(\xi), \quad x^{\prime}(1)=\beta x(\eta)
\end{array}\right.
$$

where $P:[0,1] \rightarrow \mathbb{R}^{n \times n}$ is a continuously symmetric matrix, i.e., $P^{T}(t)=P(t)$ being continuous in $t ; F:[0,1] \times \mathbb{R}^{n} \rightarrow \mathbb{R}$ is measurable in $t$ for each $x \in \mathbb{R}^{n}$ and continuously differentiable in $x$ for a.e. $t \in[0,1] ; \alpha, \beta \in \mathbb{R}, 0<\xi, \eta<1$.

Clearly, BVP (1.1) becomes a classic Sturm-Liouville BVP if $\xi \rightarrow 0$ and $\eta \rightarrow 1$.

Without loss of generality, we suppose $\xi \leq \eta$. Let $\left\{p_{i}(t)\right\}$ be the eigenvalue of $P(t)$. Assume

(H1) $0<a \leq \min _{0 \leq t \leq 1} \min _{1 \leq j \leq n} p_{j}(t) \leq \max _{0 \leq t \leq 1} \max _{1 \leq j \leq n} p_{j}(t) \leq b$;

(H2) $F(t, 0)=0, F(t,-x)=F(t, x)$, and there are $c, M>0$ such that

$$
F(t, x) \geq c|x|^{2}-M \text {. }
$$

Condition (H1) implies that $P(t)$ is an invertible matrix for each $t \in[0,1]$.

We are to show in this paper the following results via variational methods.

Theorem 1.1 Suppose assumptions $(\mathrm{H} 1)$ and $(\mathrm{H} 2)$ hold. BVP (1.1) has mn pairs of nontrivial solutions if there are $d, r>0, m \in \mathbb{N}^{+}$, such that

$$
(\nabla F(t, x), x) \leq-d|x|^{2}<-4 b m^{2} \pi^{2}|x|^{2}
$$

when $|x| \leq r$. 
Theorem 1.2 Suppose assumptions (H1) and (H2) hold. Then BVP (1.1) has infinitely many pairs of nontrivial solutions if there are $d, r>0$ and $\sigma \in(0,1)$, such that

$$
(\nabla F(t, x), x) \leq-d|x|^{1+\sigma}
$$

for $|x| \leq r$.

When condition (1.3) is replaced by a limitation condition, we have the following.

Theorem 1.3 Suppose assumptions (H1) and (H2) hold. Then BVP (1.1) has infinitely many pairs of nontrivial solutions if

$$
\lim _{|x| \rightarrow 0} \min _{0 \leq t \leq 1} \frac{(\nabla F(t, x), x)}{|x|^{2}}=-\infty .
$$

This paper is organized as follows. In Section 2, we discuss the relation of the critical point of functional $\Phi$ and the solution to BVP (1.1). In Section 3, we show that $\Phi$ satisfies the (PS)-condition. Based on Sections 2 and 3 we prove in Section 4 the theorems given above. Finally, an example is given in Section 5 to illustrate our result.

To prove the above results we need the following.

Theorem A [14] Suppose $X$ is a Banach space and $\Phi: X \rightarrow \mathbb{R}$ a continuously differentiable functional with $\Phi(0)=0$ and $\Phi$ even, bounded from below and satisfying $(P S)$-condition. If there is a set $K \subset X$ such that $K$ is homeomorphic to $S^{m-1}$ by an odd map, and $\sup _{K} \Phi<0$. Then $\Phi$ possesses at least $m$ distinct pairs of critical points.

\section{Critical point of functional and solution of BVP}

Suppose $X$ is a Banach space and $\Phi: X \rightarrow \mathbb{R}$ a differentiable functional with derivative given by

$$
\left\langle\Phi^{\prime}(u), v\right\rangle
$$

with $u, v \in X$. Let $Y \subset X$ be a closed subspace. If there is $u_{0} \in X$ such that

$$
\left\langle\Phi^{\prime}\left(u_{0}\right), v\right\rangle=0
$$

holds for all $v \in Y$, then $u_{0}$ is called a critical point of $\Phi$ with respect to $Y$. Furthermore, $u_{0}$ is called simply a critical point of $\Phi$ if $Y=X$.

Obviously, $u_{0}$ is a critical point of $\Phi$ with respect to $Y$ if it is that of $\Phi$.

Let $X=H^{1}\left([0,1], \mathbb{R}^{n}\right)$. Equip $X$ with the norm $\|\cdot\|$ defined by

$$
\|x\|=\left[\int_{0}^{1}|x(t)|^{2} \mathrm{~d} t+\int_{0}^{1}\left|x^{\prime}(t)\right|^{2} \mathrm{~d} t\right]^{\frac{1}{2}}
$$

for each $x \in X$. Then $X$ is a reflexive Banach space. Define

$$
\begin{aligned}
\Phi(x)= & \int_{0}^{1}\left[\frac{1}{2}\left(P(t) x^{\prime}(t), x^{\prime}(t)\right)+F(t, x(t))\right] \mathrm{d} t-\frac{1}{2} \delta \beta^{2}(P(1) x(\eta), x(\eta)) \\
& -\frac{1}{2} \delta \alpha^{2}(P(0) x(\xi), x(\xi))
\end{aligned}
$$


for $x \in X$, where $\delta$ is a constant.

It is easy to verify that $\Phi(0)=0$ and $\Phi(-x)=\Phi(x)$.

Furthermore, we have the following.

Lemma 2.1 If $u$ is a critical point of $\Phi(x)$, defined in (2.1) with respect to $Y=\{x \in X: x(0)=$ $-\alpha \delta x(\xi), x(1)=\beta \delta x(\eta)\}$, then $u=u(t)$ is a solution to BVP (1.1).

Proof The properties of $F$ and $P$ ensure $\Phi$ is continuously differentiable and the derivative of $\Phi$ is in the form

$$
\begin{aligned}
\left\langle\Phi^{\prime}(x), y\right\rangle= & \int_{0}^{1}\left[\left(P(t) x^{\prime}(t), y^{\prime}(t)\right)+(\nabla F(t, x(t)), y(t))\right] \mathrm{d} t \\
& -(P(1) \beta x(\eta), \delta \beta y(\eta))-(P(0) \alpha x(\xi), \delta \alpha y(\xi)),
\end{aligned}
$$

$x \in X, y \in Y$. Then the assumption that $u$ is a critical point of $\Phi$ respect to $Y$ means that

$$
\langle\Phi(u), y\rangle=0, \quad y \in Y .
$$

Let $Z=\left\{x \in C^{\infty}\left([0,1], \mathbb{R}^{n}\right): x(0)=x(\xi)=x(\eta)=x(1)=0\right\}$, then $Z \subset Y$. Furthermore, let

$$
\begin{aligned}
& Z_{1}=\{x \in Z: x(t)=0 \text { for } \xi \leq t \leq 1\}, \\
& Z_{2}=\{x \in Z: x(t)=0 \text { for } 1 \leq t \leq \xi \text { or } \eta \leq t \leq 1\}, \\
& Z_{3}=\{x \in Z: x(t)=0 \text { for } 0 \leq t \leq \eta\},
\end{aligned}
$$

and $T_{1}=[0, \xi], T_{2}=[\xi, \eta], T_{3}=[\eta, 1]$. Clearly equation (2.3) implies

$$
\langle\Phi(u), z\rangle=0, \quad z \in Z_{i}
$$

and then

$$
\begin{aligned}
0 & =\int_{T_{i}}\left[\left(P(t) u^{\prime}(t), z^{\prime}(t)\right)+(\nabla F(t, u(t)), z(t))\right] \mathrm{d} t \\
& =\int_{T_{i}}\left[-\left(\left(P(t) u^{\prime}(t)\right)^{\prime}, z(t)\right)+(\nabla F(t, u(t)), z(t))\right] \mathrm{d} t \\
& =-\int_{T_{i}}\left(\left(P(t) u^{\prime}(t)\right)^{\prime}-\nabla F(t, u(t)), z(t)\right) \mathrm{d} t .
\end{aligned}
$$

So one gets

$$
\left(P(t) u^{\prime}(t)\right)^{\prime}=\nabla F(t, u(t)), \quad \text { a.e. } t \in T_{i},
$$

since $z \in Z_{i}$ is arbitrary. Take $i=1,2,3$, then we have

$$
\left(P(t) u^{\prime}(t)\right)^{\prime}=\nabla F(t, u(t)), \quad \text { a.e. } t \in[0,1] .
$$


The equality $(2.5)$ means $\left(P u^{\prime}\right)(t)$ is continuous on $[0,1]$ and as a critical point of $\Phi$ with respect to $Y$, we have

$$
\begin{aligned}
0= & \int_{0}^{1}\left[\left(P(t) u^{\prime}(t), y^{\prime}(t)\right)+(\nabla F(t, u(t)), y(t))\right] \mathrm{d} t \\
& -\delta \beta^{2}(P(1) u(\eta), y(\eta))-\delta \alpha^{2}(P(0) u(\xi), y(\xi)) \\
= & \left.\left(P(t) u^{\prime}(t), y(t)\right)\right|_{0} ^{1}-\int_{0}^{1}\left(\left(P(t) u^{\prime}(t)\right)^{\prime}-\nabla F(t, u(t)), y(t)\right) \mathrm{d} t \\
& -\delta \beta^{2}(P(1) u(\eta), y(\eta))-\delta \alpha^{2}(P(0) u(\xi), y(\xi)) \\
= & \left(P(1) u^{\prime}(1), y(1)\right)-\left(P(0) u^{\prime}(0), y(0)\right)-(P(1) \beta u(\eta), \delta y(\eta))-(P(0) u(\xi), \delta \alpha y(\xi)),
\end{aligned}
$$

for $y \in Y$.

Especially, when $y \in Y_{1}=\{y \in Y: y(0)=y(\xi)=0\}$, one gets

$$
\begin{aligned}
0 & =\left(P(1) u^{\prime}(1), y(1)\right)-(P(1) \beta u(\eta), \delta \beta y(\eta)) \\
& =\left(P(1)\left(u^{\prime}(1)-\beta u(\eta)\right), y(1)\right)
\end{aligned}
$$

and then

$$
\begin{aligned}
& \left(P(1)\left(u^{\prime}(1)-\beta u(\eta)\right), y(1)\right)=0 . \\
& u^{\prime}(1)=\beta u(\eta),
\end{aligned}
$$

since $y(1) \in \mathbb{R}^{n}$ is arbitrary and $P(1)$ is invertible. At the same time the case $y \in Y_{2}=\{y \in$ $Y: y(\eta)=y(1)=0\}$ implies

$$
u^{\prime}(0)=\alpha u(\xi)
$$

So $u=u(t)$ is a solution to BVP (1.1).

Therefore our task is to discuss the existence of critical points of $\Phi$ in $X$.

Lemma 2.2 For each $x \in X$,

$$
|x(t)| \leq 2\|x\|
$$

Proof From

$$
\left|x_{i}(t)-\bar{x}_{i}\right| \leq \int_{0}^{1}\left|x_{i}^{\prime}(t)\right| \mathrm{d} t,
$$

one has

$$
|x(t)-\bar{x}| \leq \int_{0}^{1}\left|x^{\prime}(t)\right| \mathrm{d} t,
$$


where $\bar{x}_{i}=\int_{0}^{1} x_{i}(t) \mathrm{d} t$ and $\bar{x}=\int_{0}^{1} x(t) \mathrm{d} t$. Then

$$
\begin{aligned}
|x(t)| & \leq|\bar{x}|+\int_{0}^{1}\left|x^{\prime}(t)\right| \mathrm{d} t \\
& \leq \int_{0}^{1}|x(t)| \mathrm{d} t+\int_{0}^{1}\left|x^{\prime}(t)\right| \mathrm{d} t \\
& \leq\left[\int_{0}^{1}|x(t)|^{2} \mathrm{~d} t\right]^{\frac{1}{2}}+\left[\int_{0}^{1}\left|x^{\prime}(t)\right|^{2} \mathrm{~d} t\right]^{\frac{1}{2}} \\
& \leq 2\left[\int_{0}^{1}\left(|x(t)|^{2}+\left|x^{\prime}(t)\right|^{2}\right) \mathrm{d} t\right]^{\frac{1}{2}} \\
& =2\|x\| .
\end{aligned}
$$

\section{A lemma on the (PS)-condition}

We show at first a lemma which will be applied in the proof of our main results.

Lemma 3.1 The functional $\Phi$, defined in (2.1), satisfies the (PS)-condition if assumptions (H1)-(H2) hold.

Proof Suppose $\left\{u_{k}\right\} \subset X$ is a sequence such that $\left\{\Phi u_{k}\right\}$ is bounded and $\Phi^{\prime}\left(u_{k}\right) \rightarrow 0$ as $k \rightarrow \infty$. We are to show that there is in $\left\{u_{k}\right\}$ a subsequence which converges in $X$.

To this end, let $\theta=\min \left\{\frac{a}{2}, c\right\}>0$ and choose

$$
\delta \in\left(0, \frac{\theta}{2 b\left(\alpha^{2}+\beta^{2}\right)}\right)
$$

in the functional (2.1). Then

$$
\begin{aligned}
\Phi\left(u_{k}\right)= & \int_{0}^{1}\left[\frac{1}{2}\left(P(t) u_{k}^{\prime}(t), u_{k}^{\prime}(t)\right)+F\left(t, u_{k}(t)\right)\right] \mathrm{d} t \\
& -\frac{1}{2} \delta \beta^{2}\left(P(1) u_{k}(\eta), u_{k}(\eta)\right)-\frac{1}{2} \delta \alpha^{2}\left(P(0) u_{k}(\xi), u_{k}(\xi)\right) \\
\geq & \theta \int_{0}^{1}\left[\left|u_{k}^{\prime}(t)\right|^{2}+\left|u_{k}(t)\right|^{2}\right] \mathrm{d} t-M \\
& -\frac{1}{2} \delta\left[\beta^{2}\left(P(1) u_{k}(\eta), u_{k}(\eta)\right)+\alpha^{2}\left(P(0) u_{k}(\xi), u_{k}(\xi)\right)\right] .
\end{aligned}
$$

Notice that

$$
\begin{aligned}
\left|u_{k}(\eta)\right| & \leq\left|\bar{u}_{k}\right|+\int_{0}^{1}\left|u_{k}^{\prime}(t)\right| \mathrm{d} t \\
& \leq \int_{0}^{1}\left|u_{k}(t)\right| \mathrm{d} t+\int_{0}^{1}\left|u_{k}^{\prime}(t)\right| \mathrm{d} t \\
& \leq\left(\int_{0}^{1}\left|u_{k}(t)\right|^{2} \mathrm{~d} t\right)^{\frac{1}{2}}+\left(\int_{0}^{1}\left|u_{k}^{\prime}(t)\right|^{2} \mathrm{~d} t\right)^{\frac{1}{2}},
\end{aligned}
$$


and then

$$
\left|u_{k}(\eta)\right|^{2} \leq 2 \int_{0}^{1}\left[\left|u_{k}^{\prime}(t)\right|^{2}+\left|u_{k}(t)\right|^{2}\right] \mathrm{d} t=2\left\|u_{k}\right\|^{2} .
$$

Similarly,

$$
\left|u_{k}(\xi)\right|^{2} \leq 2 \int_{0}^{1}\left[\left|u_{k}^{\prime}(t)\right|^{2}+\left|u_{k}(t)\right|^{2}\right] \mathrm{d} t=2\left\|u_{k}\right\|^{2} .
$$

Therefore, we have

$$
\begin{aligned}
\Phi\left(u_{k}\right) & \geq \theta\left\|u_{k}\right\|^{2}-\frac{1}{4} \delta b\left(\alpha^{2}+\beta^{2}\right)\left\|u_{k}\right\|^{2}-M \\
& =\frac{1}{2} \theta\left\|u_{k}\right\|^{2}-M,
\end{aligned}
$$

which implies $\left\{u_{k}\right\}$ is bounded in $X$. Going, if necessary, to a subsequence, we assume that $u_{k} \rightarrow u$ in $X$ and $u_{k} \rightarrow u$ in $C\left([0,1], \mathbb{R}^{n}\right)$. Then

$$
\left\langle\Phi^{\prime}\left(u_{k}\right)-\Phi^{\prime}(u), u_{k}-u\right\rangle \rightarrow 0 \quad \text { as } k \rightarrow \infty .
$$

Using (2.2) and assumptions (H1)-(H2), we have

$$
\begin{aligned}
& \left\langle\Phi^{\prime}\left(u_{k}\right)-\Phi^{\prime}(u), u_{k}-u\right\rangle \\
& =\int_{0}^{1}\left[\left(P(t)\left(u_{k}^{\prime}(t)-u_{k}(t)\right), u_{k}^{\prime}(t)-u_{k}(t)\right)\right. \\
& \left.\quad+\left(\nabla F\left(t, u_{k}(t)\right)-\nabla F(t, u(t)), u_{k}(t)-u(t)\right)\right] \mathrm{d} t \\
& \quad-\delta\left[\left(P(1) \beta\left(u_{k}(\eta)-u(\eta)\right), \beta\left(u_{k}(\eta)-u(\eta)\right)\right)\right. \\
& \left.\quad+\left(P(0) \alpha\left(u_{k}(\xi)-u(\xi)\right), \alpha\left(u_{k}(\xi)-u(\xi)\right)\right)\right] \\
& \leq a \int_{0}^{1}\left|u_{k}^{\prime}(t)-u^{\prime}(t)\right|^{2} \mathrm{~d} t+\int_{0}^{1}\left(\nabla F\left(t, u_{k}(t)\right)-\nabla F(t, u(t)), u_{k}(t)-u(t)\right) \mathrm{d} t \\
& \quad-\delta b\left[\beta^{2}\left|u_{k}(\eta)-u(\eta)\right|^{2}+\alpha^{2}\left|u_{k}(\xi)-u(\xi)\right|^{2}\right] .
\end{aligned}
$$

The fact that $u_{k} \rightarrow u$ in $C\left([0,1], \mathbb{R}^{n}\right)$ implies

$$
\begin{aligned}
& \int_{0}^{1}\left(\nabla F\left(t, u_{k}(t)\right)-\nabla F(t, u(t)), u_{k}(t)-u(t)\right) \mathrm{d} t \rightarrow 0, \\
& \left|u_{k}(\eta)-u(\eta)\right| \rightarrow 0, \quad\left|u_{k}(\xi)-u(\xi)\right| \rightarrow 0, \\
& \int_{0}^{1}\left|u_{k}(t)-u(t)\right|^{2} \mathrm{~d} t \rightarrow \infty,
\end{aligned}
$$

as $k \rightarrow \infty$. Then from (3.3) and (3.4) we get

$$
\int_{0}^{1}\left|u_{k}^{\prime}(t)-u^{\prime}(t)\right|^{2} \mathrm{~d} t \rightarrow 0 \quad \text { as } k \rightarrow \infty .
$$


Therefore, we have

$$
u_{k} \rightarrow u \quad \text { in } X
$$

Then $\Phi$ satisfies the (PS)-condition.

\section{Proof of theorems}

Proof of Theorem 1.1 First we show that $\Phi(x)$ is bounded from below.

From the definition of $\Phi$ in (2.1), one has

$$
\begin{aligned}
\Phi(x) & \geq \theta \int_{0}^{1}\left[\left|x^{\prime}(t)\right|^{2}+|x(t)|^{2}\right] \mathrm{d} t-M-\frac{1}{2} \delta b\left(\beta^{2}|x(\eta)|^{2}+\alpha^{2}|x(\xi)|^{2}\right) \\
& =\theta\|x\|^{2}-\frac{1}{2} \delta b\left(\beta^{2}|x(\eta)|^{2}+\alpha^{2}|x(\xi)|^{2}\right)-M \\
& \geq \theta\|x\|^{2}-\frac{1}{2} \delta b\left(\beta^{2}+\alpha^{2}\right)\|x\|^{2}-M \\
& \geq \frac{1}{2} \theta\|x\|^{2}-M,
\end{aligned}
$$

which implies that $\Phi(x)$ is bounded from below.

Second, we prove the existence of a set $K \subset X$ such that $K$ is homeomorphic to $S^{m n-1}$ by an odd map, and $\sup _{K} \Phi<0$.

To this end we choose the linear space $X_{m n}$ in the following way.

Let $\left\{e_{i}\right\}$ be the orthogonal basis of $\mathbb{R}^{n}$. As Banach space $H^{1}$ is a subspace of $L^{1}\left([0,1], \mathbb{R}^{n}\right)$, its element can be expressed in the form

$$
x(t)=a_{0}+\sum_{k=1}^{\infty}\left(\cos 2 k \pi t \cdot a_{k}+\sin 2 k \pi t \cdot b_{k}\right), \quad 0<t<1,
$$

where $a_{0}, a_{k}, b_{k} \in \mathbb{R}^{n}$. In this case, let

$$
x(0)=x\left(0^{+}\right), \quad x(1)=x\left(1^{-}\right),
$$

and

$$
\begin{aligned}
& x^{\prime}(0)=\lim _{t \rightarrow 0^{+}} \frac{x(t)-x(0)}{t}=\lim _{t \rightarrow 0^{+}} \frac{1}{t}\left(x(t)-x\left(0^{+}\right)\right), \\
& x^{\prime}(1)=\lim _{t \rightarrow 1^{-}} \frac{x(1)-x(t)}{1-t}=\lim _{t \rightarrow 1^{-}} \frac{1}{1-t}\left(x\left(1^{-}\right)-x(t)\right) .
\end{aligned}
$$

Let $X_{m n}=\left\{x(t)=\sum_{k=1}^{m} \sin 2 k \pi t \cdot b_{k}, b_{k} \in \mathbb{R}^{n}\right\}$. Then $\operatorname{dim} X_{m n}=m n$. For a function $x(t)=$ $\sum_{k=1}^{m} \sin 2 k \pi t \cdot b_{k}$, one gets

$$
x^{\prime}(t)=\sum_{k=1}^{m} 2 k \pi \cos 2 k \pi t \cdot b_{k}
$$


and

$$
\begin{aligned}
& \int_{0}^{1}|x(t)|^{2} \mathrm{~d} t=\frac{1}{2} \sum_{k=1}^{m}\left|b_{k}\right|^{2} \\
& \int_{0}^{1}\left|x^{\prime}(t)\right|^{2} \mathrm{~d} t=\frac{1}{2} \sum_{k=1}^{m}(2 k \pi)^{2}\left|b_{k}\right|^{2} \leq 4 m^{2} \pi^{2} \int_{0}^{1}|x(t)|^{2} \mathrm{~d} t .
\end{aligned}
$$

It follows that

$$
\|x\|^{2}=\frac{1}{2} \sum_{k=1}^{m}\left(1+4 k^{2} \pi^{2}\right)\left|b_{k}\right|^{2} \leq \frac{1}{2}\left(1+4 m^{2} \pi^{2}\right) \sum_{k=1}^{m}\left|b_{k}\right|^{2}
$$

and

$$
\frac{1}{1+4 m^{2} \pi^{2}}\|x\|^{2} \leq \int_{0}^{1}|x(t)|^{2} \mathrm{~d} t \leq \frac{1}{1+4 \pi^{2}}\|x\|^{2} .
$$

Now choose $K=\left\{x \in X_{m n}, \sum_{k=1}^{m}\left|b_{k}\right|^{2}=\frac{1}{m} r^{2}\right\}$. Obviously, $K$ is closed in $X$ with $\operatorname{dim} K=$ $m n-1$. Furthermore, for each $x \in K$,

$$
\begin{aligned}
& \Phi(x) \leq \int_{0}^{1}\left[\frac{b}{2}\left|x^{\prime}(t)\right|^{2}+F(t, x(t))\right] \mathrm{d} t \\
& |x(t)| \leq \sum_{k=1}^{m}\left|b_{k}\right| \leq \sqrt{m}\left(\sum_{k=1}^{m}\left|b_{k}\right|^{2}\right)^{\frac{1}{2}}=r^{2} .
\end{aligned}
$$

At the same time we have, from (4.3),

$$
\begin{aligned}
F(t, x(t)) & =F(t, x(t))-F(t, 0) \\
& =\int_{0}^{1}(\nabla F(t, s x(t)), x(t)) \mathrm{d} s \\
& =\int_{0}^{1} \frac{1}{s}(\nabla F(t, s x(t)), s x(t)) \mathrm{d} s \\
& <-4 b m^{2} \pi^{2} \int_{0}^{1} s|x(t)|^{2} \mathrm{~d} s \\
& =-2 b m^{2} \pi^{2}|x(t)|^{2},
\end{aligned}
$$

which yields

$$
\int_{0}^{1} F(t, x(t)) \mathrm{d} t<-2 b m^{2} \pi^{2} \int_{0}^{1}|x(t)|^{2} \mathrm{~d} t
$$

and

$$
\begin{aligned}
\Phi(x) & <\frac{b}{2} \int_{0}^{1}\left|x^{\prime}(t)\right|^{2} \mathrm{~d} t-2 b m^{2} \pi^{2} \int_{0}^{1}|x(t)|^{2} \mathrm{~d} t \\
& \leq 2 b m^{2} \pi^{2} \int_{0}^{1}|x(t)|^{2} \mathrm{~d} t-2 b m^{2} \pi^{2} \int_{0}^{1}|x(t)|^{2} \mathrm{~d} t \\
& =0
\end{aligned}
$$


Then we have

$$
\sup _{K} \Phi<0 .
$$

Finally, define the odd mapping $G: K \rightarrow S^{m n-1}$ in the following way. For a function $x \in K$ with the expression

$$
x(t)=\sum_{k=1}^{m} \sin 2 k \pi t \cdot b_{k},
$$

let $x(t) \mapsto G(x)=\left(\frac{1}{\rho} b_{1}, \frac{1}{\rho} b_{2}, \ldots, \frac{1}{\rho} b_{m}\right)$, where $\rho=\left(\sum_{k=1}^{m}\left|b_{k}\right|^{2}\right)^{\frac{1}{2}}$. Then $G$ is a homeomorphism between $K$ and $S^{m-1}$. It is clear that $G$ is an odd mapping.

Then Theorem A gives the conclusion of Theorem 1.1.

Proof of Theorem 1.2 It suffices to show that for any $m \in \mathbb{N}$, condition (1.3) implies that there is $\hat{r}>0$ such that the condition holds for $r \in(0, \hat{r})$.

In fact, from $\lim _{|x| \rightarrow 0^{+}}|x|^{1+\sigma} /|x|^{2}=+\infty$, we know that there is $\hat{r} \in(0, r)$ such that

$$
d|x|^{1+\sigma}>4 b m^{2} \pi^{2}|x(t)|^{2}, \quad 0<|x| \leq \hat{r} .
$$

In this case, we have

$$
(\nabla F(t, x), x) \leq-d|x|^{1+\sigma}<-4 b m^{2} \pi^{2}|x(t)|^{2}, \quad 0<|x| \leq \hat{r},
$$

which implies, by Theorem 1.1, that BVP(1.1) has at least $m n$ pairs of distinct nontrivial solutions.

Proof of Theorem 1.3 Condition (1.4) implies that for any $m \in \mathbb{N}$ there is $r>0$ such that

$$
(\nabla F(t, x), x) \leq-4 b(m+1)^{2} \pi^{2}|x(t)|^{2}<-4 b m^{2} \pi^{2}|x|^{2}, \quad 0<|x| \leq r .
$$

Then the conclusion comes from Theorem 1.1.

\section{Example}

Example 5.1 Suppose $x_{1}, x_{2}:(0,1) \rightarrow \mathbb{R}$. Then the BVP

$$
\left\{\begin{array}{l}
5 x_{1}^{\prime \prime}+3 x_{2}^{\prime \prime}=\left[-\left(1+\sin ^{2} t\right)\left(x_{1}^{2}+2 x_{2}^{2}\right)^{-\frac{1}{4}}+6\left(1+t^{2}\right)\left(2 x_{1}^{2}+x_{2}^{2}\right)^{\frac{1}{2}}\right] x_{1}, \\
3 x_{1}^{\prime \prime}+5 x_{2}^{\prime \prime}=\left[-2\left(1+\sin ^{2} t\right)\left(x_{1}^{2}+2 x_{2}^{2}\right)^{-\frac{1}{4}}+3\left(1+t^{2}\right)\left(2 x_{1}^{2}+x_{2}^{2}\right)^{\frac{1}{2}}\right] x_{2} \\
x_{1}^{\prime}(0)=3 x_{1}\left(\frac{1}{4}\right), \quad x_{1}^{\prime}(1)=\frac{1}{3} x_{1}\left(\frac{3}{4}\right) \\
x_{2}^{\prime}(0)=3 x_{1}\left(\frac{1}{4}\right), \quad x_{2}^{\prime}(1)=\frac{1}{3} x_{2}\left(\frac{3}{4}\right)
\end{array}\right.
$$

has infinitely many solutions.

Proof Let $M(t)=\left[\begin{array}{ll}5 & 3 \\ 3 & 5\end{array}\right], \alpha=3, \beta=\frac{1}{3}, \xi=\frac{1}{4}, \eta=\frac{3}{4}, x=\left(\begin{array}{l}x_{1} \\ x_{2}\end{array}\right), F(t, x)=-\frac{2}{3}\left(1+\sin ^{2} t\right)\left(x_{1}^{2}+\right.$ $\left.2 x_{2}^{2}\right)^{\frac{3}{4}}+\left(1+t^{2}\right)\left(2 x_{1}^{2}+x_{2}^{2}\right)^{\frac{3}{2}}$, then BVP (5.1) is a special case of BVP (1.1) with $n=2$. 
Obviously, the eigenvalues of $M$ are 2 and 8 , which means

$0<a=2, \quad b=8$.

On the other hand, we have

$$
\begin{aligned}
& \lim _{|x| \rightarrow \infty} F(t, x)=+\infty, \quad F(t, 0)=0, \quad F(t,-x)=F(t, x), \\
& \lim _{|x| \rightarrow \infty} \frac{(\nabla F(t, x), x)}{|x|^{2}}=-\infty,
\end{aligned}
$$

and

$$
\begin{aligned}
F(t, x(t)) & \geq-\frac{8}{3}\left(x_{1}^{2}+x_{2}^{2}\right)^{\frac{3}{4}}+\left(x_{1}^{2}+x_{2}^{2}\right)^{\frac{3}{2}} \\
& \geq-\frac{8}{3}\left(1+x_{1}^{2}+x_{2}^{2}\right)+3\left(x_{1}^{2}+x_{2}^{2}\right)-27 \\
& =\frac{1}{3}\left(x_{1}^{2}+x_{2}^{2}\right)-\frac{89}{3} .
\end{aligned}
$$

Let $c=\frac{1}{3}$ and $M=\frac{89}{3}$. Then Theorem 1.3 gives the conclusion.

\section{Competing interests}

The authors declare that they have no competing interests.

\section{Authors' contributions}

All authors contributed equally to the writing of this paper. All authors read and approved the final manuscript.

\section{Author details}

'Department of Mathematics, Beijing Institute of Technology, Beijing, 100081, P.R. China. ${ }^{2}$ Department of Mathematics, University of Science and Technology Beijing, Beijing, 100083, P.R. China.

\section{Acknowledgements}

This work is supported by Beijing Higher Education Young Elite Teacher Project Grant No. YETP0388, and Specialized Research Fund for the Doctoral Program of Higher Education (SRFDP), Grant No. 20120006120007. The authors would like to thank the referee for their valuable comments and suggestions on the original manuscript.

Received: 2 December 2015 Accepted: 14 February 2016 Published online: 25 March 2016

\section{References}

1. Bonanno, G, Livra, R: Multiplicity theorems for the Dirichlet problem involving the p-Laplacian. Nonlinear Anal. 54, 1-7 (2003)

2. Bonanno, G: Multiple critical points theorems without the Palais-Smale condition. J. Math. Anal. Appl. 299, 600-614 (2004)

3. Tian, Y, Ge, W: Multiple solutions for a second-order Sturm-Liouville boundary value problem. Taiwan. J. Math. 11(4), 975-988 (2007)

4. Tian, Y, Ge, W: Multiple positive solutions for periodic boundary value problem via variational methods. Tamkang J. Math. 39(2), 111-119(2008)

5. Tian, Y, Ge, W: Second-order Sturm-Liouville boundary value problem involving the one-dimensional p-Laplacian. Rocky Mt. J. Math. 38(1), 309-324 (2008)

6. Nieto, JJ, O'Regan, D: Variational approach to impulsive differential equations. Nonlinear Anal., Real World Appl. 10(2), 680-690 (2009)

7. Nieto, JJ: Variational formulation of a damped Dirichlet impulsive problem. Appl. Math. Lett. 23(8), 940-942 (2010)

8. Tian, Y, Ge, W: Applications of variational methods to boundary-value problem for impulsive differential equations. Proc. Edinb. Math. Soc. 51, 509-527 (2008)

9. Tian, Y, Ge, W: Multiple solutions of impulsive Sturm-Liouville boundary value problem via lower and upper solutions and variational methods. J. Math. Anal. Appl. 387(2), 475-489 (2012)

10. Mawhin, J, Willem, M: Critical Point Theory and Hamiltonian Systems. Applied Mathematical Sciences, vol. 74. Springer, New York (1989)

11. Tian, Y, Ge, W: Periodic solutions of non-autonomous second-order systems with a p-Laplacian. Nonlinear Anal., Theory Methods Appl. 66(1), 192-203 (2007) 
12. Graef, JR, Heidarkhani, S, Kong, L: A critical points approach to multiplicity results for multi-point boundary value problems. Appl. Anal. 90(12), 1909-1925 (2001)

13. Ricceri, B: A three critical points theorem revisited. Nonlinear Anal., Theory Methods Appl. 70(9), 3084-3089 (2009)

14. Clark, D: A variant of the Lusternik-Schnirelman theory. Indiana Univ. Math. J. 22, 65-74 (1972)

Submit your manuscript to a SpringerOpen ${ }^{\circ}$ journal and benefit from:

- Convenient online submission

- Rigorous peer review

- Immediate publication on acceptance

- Open access: articles freely available online

- High visibility within the field

Retaining the copyright to your article

Submit your next manuscript at $\boldsymbol{s p r i n g e r o p e n . c o m ~}$ 